\title{
Grid Approximation Based Inductive Charger Deployment Technique in Wireless Sensor Networks
}

\author{
Fariha Tasmin Jaigirdar \\ Dept. of Computer Science \\ Stamford University, Bangladesh \\ Dhaka, Bangladesh. \\ farihajaigirdar@yahoo.com
}

\author{
Mohammad Mahfuzul Islam \\ Dept. of Computer Science \& \\ Engineering \\ Bangladesh University of \\ Engineering \& Technology (BUET) \\ Dhaka, Bangladesh. \\ mahfuz@cse.buet.ac.bd
}

\author{
Sikder Rezwanul Huq \\ Dept. of Computer Science \& \\ Engineering \\ Islamic University of Technology \\ (IUT) \\ Dhaka, Bangladesh. \\ rezwan_cit_2005@yahoo.com
}

\begin{abstract}
Ensuring sufficient power in a sensor node is a challenging problem now-a-days to provide required level of security and data processing capability demanded by various applications scampered in a wireless sensor network. The size of sensor nodes and the limitations of battery technologies do not allow inclusion of high energy in a sensor. Recent technologies suggest that the deployment of inductive charger can solve the power problem of sensor nodes by recharging the batteries of sensors in a complex and sensitive environment. This paper provides a novel grid approximation algorithm for efficient and low cost deployment of inductive charger so that the minimum number of chargers along with their placement locations can charge all the sensors of the network. The algorithm proposed in this paper is a generalized one and can also be used in various applications including the measurement of network security strength by estimating the minimum number of malicious nodes that can destroy the communication of all the sensors. Experimental results show the effectiveness of the proposed algorithm and impacts of the different parameters used in it on the performance measures.
\end{abstract}

Keywords- wireless sensor network; energy efficiency; network security; grid approximation; inductive charger.

\section{INTRODUCTION}

With the rapid development of computing and communication technologies, Wireless Sensor Networks (WSNs) are now being used in various applications including environment monitoring, healthcare, complex and sensitive surveillance systems and military purposes. These applications, however, demands high level of system security and longer lifespan of the sensors. WSNs are self-organized ad-hoc networks consisting of a large number of tiny sensor nodes having small sized low capacity batteries and able to communicate over wireless media. The low power battery limits a sensor node in providing a desired level of processing and buffering capabilities and hence refrains from providing necessary securities [1] [2] [3].

Large sized batteries and expensive sensors can mitigate the low energy problem of WSNs. However, these solutions are not feasible because of the nature of WSN deployment especially when it is deployed in an unfriendly environment like battlefield or underwater investigation. On the other hand, the sensors of a WSN run unassisted for a long time and they can neither be disposable nor its battery can be replaced or recharged. Another possibility of dealing with the low energy problem is to deploy new external sensors in the network region periodically to keep the network alive. Some locations of the network (e.g., deployment of sensor nodes inside a machine or in a dangerous/unreachable forest, etc) do not even permit to deployment of new sensors even if cost is ignored. Thus, recharging from a distance is the only effective and generalized approach for dealing with the power problem of WSNs. Many research works [4][5][6] have been proposed to keep WSNs alive or prolong the network lifetime by recharging the batteries using the distance based recharging technique, which is also called the inductive charging technology [7]. This technology is convenient, safe, efficient and green [8].

There are different ways of recharging the sensors inductively. Some research works have been proposed to recharge sensor nodes by extracting energy directly from the deployment environment. These are called "scavenging" techniques [4]. The scavenging techniques recharge the sensors by collecting energies from solar power [9], kinetic energy [10], floor vibration [11], acoustic noise and so on. Due to the requirement of large exposure area of sensing devices, these techniques are not feasible to accommodate in tiny sensors. Moreover, the power generated by using these techniques is not sufficient enough for sustaining the regular operation of sensor nodes. Recent research works investigate that mobile nodes can deliver power to the sensors inductively (i.e., cordlessly) by creating an electromagnetic channel [8]. This amount of power supplied by this technology is sufficient to meet the power requirement of TV, laptop, mobile phone, digital camera, PDA and so on[8]. Sensors nodes can collect sufficient powers from the infrequent visit of active mobile nodes and hence prolong the WSN lifetime.

Efficient and cost-effective deployment of active mobile nodes is one of the key problems for introducing inductive sensor charging technique. To the best of our knowledge, there 
is no existing technique to solve this problem. In this paper, we propose a solution by finding the minimum number of active mobile nodes (also known as charging nodes (i.e., charger)) along with their locations, which can charge all the sensors of a network. Here, our goal is to maintain continuous energy supply to the sensing nodes by placing the chargers in appropriate locations that will gain the efficiency of the work and also for ensuring the cost effectiveness, our approach will find the minimum number of chargers as well. The solution seems close to that of the coverage problem, but in real sense it is totally different. The coverage problem schemes are mainly of two types: area coverage and target or point coverage. The area coverage schemes explore the solution to cover the entire area of a WSN, while point coverage schemes, a special case of area coverage problem, focus on determining the exact position of sensor nodes to provide efficient coverage application for a limited number of targets [12]. Finding the optimum location of active nodes is an NP-hard problem. Therefore, our solution focuses on finding out the best locations of placing active nodes by exploiting and merging the techniques of grid approximation [13], minimum set cover problem [14] and the greedy approach [15]. The experimental results clearly reveal that, by setting appropriate parameters, the proposed solution can efficiently find out the best locations of the chargers.

The rest of the paper is organized as follows: Section 2 discusses the background and related works of the problem, while the details of the proposed inductive charger deployment problem have been given in Section 3. The experimental setup, parameters and results along with comparative analysis has been given in Section 4. Some concluding remarks and future works are given in Section 5.

\section{BACKGROUND AND RELATED WORKS}

To the very best of our knowledge, the charger deployment strategy that we approach here is unique and does not directly relate to any other work. Moreover, to better understand the concepts and clarify the novelty of our work we discuss different coverage problems in this section. As we are concerning with the energy issue, different power factor related works are also discussed in this section. The coverage problem deals with the quality of service (QoS) that can be provided by a particular sensor network. The term coverage means how well a sensor network is monitored or tracked by the sensors. As the purpose of WSN is to collect relevant data for processing, it can be done properly by covering the overall network to achieve the required goal. Many research works [16] [17] have been proposed in this area and researchers define the problems from different angles and also gave different design view.

In this part, we have studied different coverage approaches and classified them considering the coverage concept. As stated before, the two main parts of coverage problems along with some design choices [18] have added here for better understanding the problem and its applications.
- Sensor Deployment Method: Deterministic versus random. A deterministic sensor placement is the method in which the location and the number of sensors needed are predetermined and is feasible in friendly, previously organized and accessible environments. But in the scenario of deployment in remote and inhospitable areas, deterministic node placement can be impractical for some extent and the solution is random sensor distribution where having knowledge about the number and location of sensor nodes will be ignored.

- Communication Range: An important factor that relates to connectivity is communication range, which can be equal or not equal to the sensing range.

- Additional Critical Requirements: Energyefficiency, connectivity and fault tolerance.

- Algorithm Characteristics: centralized versus distributed/localized.

The most studied coverage problem is the area coverage problem. As an important research issue many researchers have been studied extensively on this topic and different sensor network applications have revealed a new era to solve their area coverage problem in different scenarios by varying design choices and other factors. To better understand the area coverage scheme and also how it differs from our work, here we have shown a brief discussion on area coverage.

As related to our energy efficiency issue, we have discussed here the power efficient coverage with random deployment. In the previous section, we have already discussed about power factors and consideration in WSNs and we have come to know that replacing the sensor node's battery is not feasible in many applications, so, approaches that has power conserve facility, are highly desirable. The works in [19] and [20] consider a large amount of sensors, deployed randomly for area monitoring. The contribution in [19] explains why energy is a factor of consideration in sensor network. In these papers, the goal is to achieve an energyefficient design that maintains area coverage. As the number of sensors deployed is greater than the optimum required to perform the monitoring task, the solution proposed is to divide the sensor nodes into disjoint sets, such that every set can individually perform the area monitoring tasks. These set areas are then activated successively to perform the area monitoring task, and while the current sensor set is active, all other nodes are in a low-energy sleep mode to sustain the battery lifetime for further activations. The goal of this approach is to determine a maximum number of disjoint sets, as this has a direct impact on the network lifetime.

Another special case issue of area coverage is point coverage. An example of it showed in [21] has military applicability. In this paper the authors addressed the problem of energy efficiency in wireless sensor applications for surveillance of a set of limited number of targets with known locations. Here, a large number of sensors are dispersed 
randomly in close proximity to the monitor the targets; and finally the sensors send the monitored information to a central processing node. The objective is that every target must be monitored at all times by at least one sensor and on the road to reach the required goal the authors have given a solution of preparing disjoint sets of sensor nodes and maintaining maximum number of such sets to be activated successively. Here, the authors prove that the disjoint set coverage problem is NP-complete and propose an efficient heuristic for set covers computation using a mixed integer programming formulation.

One more work of point coverage proposed in [22], where energy efficiency still maintained by covering targets with sensors. In this paper the author model the solution as the maximum set cover problem and design two heuristics that efficiently compute the sets, using linear programming and greedy approach. Here, in maximum set cover (MSC) definition, $\mathrm{C}$ denotes the set of sensors and $\mathrm{R}$ the set of targets, such that each sensor covers a subset of targets. In the greedy approach, at each step, a critical target is selected to be covered. This can be, for example, the target most sparsely covered, both in terms of sensors as well as the residual energy of those sensors. Upon selecting the critical target, the heuristic selects the sensor with the greatest contribution that covers the critical target. Once a sensor has been selected it is added to the current set cover and all additionally covered targets are removed from the TARGETS set, which contains the targets that still have to be covered by the current set cover. When all targets are covered, the new set cover was formed. Here simulation results are presented to verify the approaches.

\section{OUR PROPOSED GRID APPROXIMATION STRATEGY}

Our objective here is to strategically place the mobile chargers in spite of deploy them blindly to charge all the nodes in the network using inductive charging technique. Here, the word "strategically" means the way of deployment of the chargers that we have showed here to be best. Actually, a blind deployment strategy may show better result in some scenarios, but it will not always happen, and not guaranteed to be any time, moreover, it would take away the solution to an infinite searching that should not be our goal. By our proposed deployment strategy, it would be possible for us to find the best locations and as well as the minimum amount of charging nodes or chargers to charge all the sensor nodes in the network and also we would gain the efficiency and cost effectiveness of the deployment methodology and reach the required goal as well.

\section{A. The Strategy}

Here to cover (i.e., to charge) all the sensor nodes in the network, at first the search space for the solution will be identified. Obviously an infinite searching can achieve the optimum solution. However, to devise any practical implement able solution the search space needs to be reduced and made finite at the cost of optimality.
Our approach for making search space finite is the well known grid approximation technique. In this methodology, we will divide the entire network deployment area into grid points. The sensing nodes at first will be placed randomly over this deployment area. Then from every grid points, all the deployed sensing node's distances will be calculated by using the famous distance calculation equation. Finally these distances will be compared with the transmission range of the nodes. Note that a sensing node may be within the range of several grid points. As the target is to place the mobile chargers in grid points only so that all the sensor nodes can be charged and finding the minimum number of such grid points, hence the problem can be easily mapped to a minimum set cover problem, because, in minimum set cover problem the target is to find the minimum number of sets to cover every element and that is actually our required goal. Greedily, we can at first place a mobile charger to the grid point which can cover maximum number of sensing nodes, then remove all the covered nodes and reapply the greedy approach until all the sensing nodes are being charged. Here, we use the greedy approach, as algorithms of this type often leads to very efficient and simple solution [15] and that tends us to fulfill our desired solution. Upon finding the first grid point that will cover the maximum number of sensing nodes in its transmission range, we will keep the location of that point also to find out the position of the first charger and processing in this way we will find all the chargers position and the number of chargers needed to charge all the sensing nodes in the network using inductive charging technique.

\section{B. Mathematical Representation}

Given a WSN of $N$ sensing nodes deployed in a two dimensional field of size $D \times D$, where the transmission range of every node is considered as $R$. Here, we assume that we have full knowledge about the network topology in the way that each node has a low-power Global Position System (GPS) receiver, which provides the position information of the node itself. If GPS is not available, the distance between neighboring nodes can be estimated on the basis of incoming signal strengths. To cover a wireless node $v(\mathrm{i}, \mathrm{j}$ ), a mobile node or charger $j(\mathrm{x}, \mathrm{y})$ should be placed inside an $\mathrm{R}$ radius circle centered at $v$. Here, at first the distance between $j$ and $v$ will be calculated using Eq. (1)

$$
\operatorname{dis}(j, v)=\sqrt{ }\left((x-i)^{2}+(y-j)^{2}\right) .
$$

Now, this value of distance will be compared with the transmission range of nodes, i.e., if dis $(\mathrm{j}, \mathrm{v})<\mathrm{R}$, we can say that by placing a charging node or charger at grid point $j(\mathrm{x}, \mathrm{y})$ we can charge the node $v(\mathrm{i}, \mathrm{j})$. We will continue on finding the distance of all the sensor nodes from all the grid points in the total networking topology and hence we will apply our greedy approach, that is, upon finding the grid point that will have maximum number of sensing nodes in its transmission range, we will place our first charger to that point and remove the sensors (that have been charged by that point) from the entire set of sensing nodes by marking them charged and 
reapply the greedy method to find the minimum number of chargers needed to charge all the sensing nodes in the network. Here, our goal is to find minimum number of grid point $\mathrm{j}(\mathrm{x}, \mathrm{y})$ where to place the chargers to charge all the sensors $N$ in the network. So, it can be easily expressed by the following equation, i.e., Eq. (2).

$$
\min (j(x, y))=V(\text { all the nodes, } N)
$$

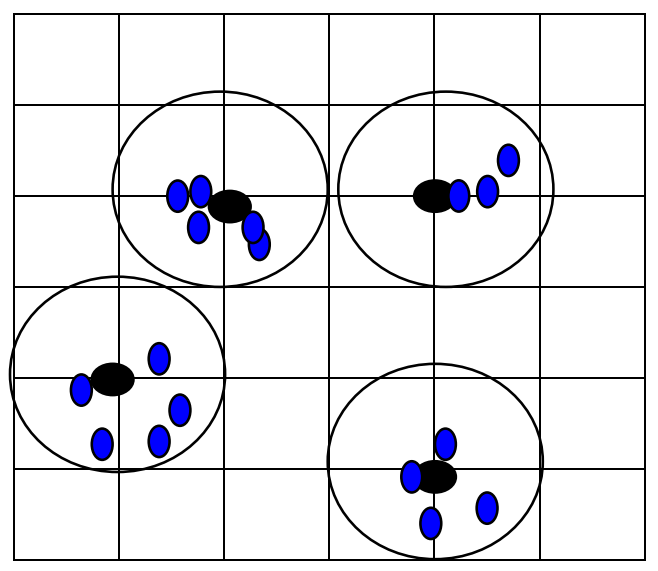

Figure 1. Minimum number of chargers needed to charge all the nodes in the network using Grid Approximation strategy.

The scenario can be understood easily from Fig. 1. In the figure, the blue circles represent the sensor nodes and the black circles represent the mobile charging nodes or chargers that we will deploy strategically by using our Grid Approximation strategy. The outline of the black nodes represents their transmission range. Here, our target is to deliver continuous energy supply to the sensing nodes and we have decided to use inductive charging technique for this purpose. If we place the external chargers which we call active mobile nodes blindly to charge all the nodes in the network it is possible that we would need seventeen chargers as in this case to charge a sensor we would need one charger. On the contrary, we can see from Fig. 1, if we use the Grid Approximation technique, to charge all the seventeen sensing nodes, we need only four chargers and it is also possible that the number of chargers will be lessened for different network topology. Here, the positions of the chargers are $(1,2),(2,4)$, $(4,1)$ and $(4,4)$.

Here, in Grid Approximation technique, we have taken unit distance between the grid points and that we call step size 1. Actually a unit distanced approximation technique will give a best result of our problem but the processing steps and time will increase, which is not desirable. So we tried to increase the distance between the grid points by a pruning strategy, which will guarantee not to leave any points inside the working area and also reduce the processing steps and time as it will help in solving the brute force technique that we stated before. To cover a square shaped area, which we use here just to represent a small structural view of a grid, and here it is recommended that no small point can be missed, we can find our desired step size value or maximum distance between the grid points by placing four circles in four corners. In the Fig. 2 , we can see that if the sum of the radius of two circles (that are placed in opposite corner of a square) is exactly equals the diagonal measurement of the square area, and then it is guaranteed not to leave any point inside the area. Moreover, it is the maximum distance between the grid points as in this scenario the maximum value of the diagonal can be $2 \mathrm{R}$, where $\mathrm{R}$ is the radius of the circles. In Fig. 2, the maximum value of the diagonal PQ can be 2R, so using Pythagoras theorem [23], we can find our desired value and the value is derived in Eq. (3). Here we consider the value of $\mathrm{P}$ to $\mathrm{X}$-axis and $\mathrm{P}$ to $\mathrm{Y}$-axis is same and that is $\mathrm{X}$, so by Pythagoras theorem we can write, $X^{2}+X^{2}=(2 R)^{2}$. So,

$$
X=\sqrt{ } 2 R .
$$

Hence, the highest step size or maximum distance between two grid points can be $\sqrt{2} \mathrm{R}$, without leaving any point of interest within the network area untouched.

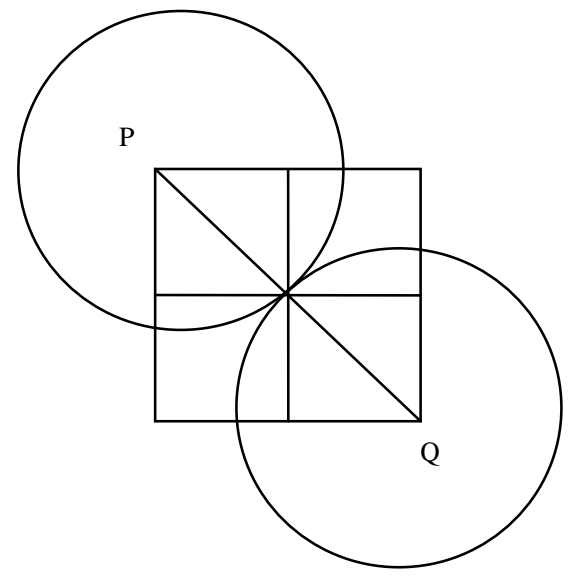

Figure 2. The maximum distance between two grid points to cover all sensors.

\section{Algorithm}

We have placed our proposed Grid Approximation algorithm in Fig. 3. The different parameters used in the algorithms are number of nodes, step size value, transmission range, maximum value in $\mathrm{X}$-axis and $\mathrm{Y}$-axis. Here, we start by considering that all the sensing nodes are uncharged at first iteration and the algorithm will stop its iteration upon charge all the sensing nodes. In this algorithm, we have made a comparison between the distance and transmission range or radius of sensing nodes. We have counted the number of neighbors a grid point has by considering the transmission range, and hence our algorithm's goal is to find the grid point that has highest number of sensing nodes as its neighbors. This is the point that we call high_freq $(\mathrm{x}, \mathrm{y})$ in our algorithm. Upon finding the point we will place our first charger at that point 
and remove all its neighbors of that point marking as being charged and will continue the algorithm with the sensing nodes that have not been charged yet. We reapply the strategy until all the sensing nodes are being charged. So, finally we get the minimum number of chargers and their locations that we need to charge all the sensing nodes in the network.

Algorithm Grid (NN, R, XMAX, YMAX, $\Delta$ )
//NN=Number of sensor nodes,
//num_not_charged_yet=number of not_charged
//nodes, high_freq=highest number of nodes covered,
//high_freq_X, high_freq_y=represents the coordinates
//of highest frequency point, i.e., the points that have
//covered maximum number of nodes in its
//transmission range, XMAX=maximum dimension in
//x-axis, YMAX=maximum dimension in y-axis,
//R=transmission range or radius, $\Delta=$ distance between
//the grid points.
num_not_charged_yet=NN
while num_not_charged_yet $>0$ do
high_freq:=0
for $\mathrm{i}:=0$ to YMAX step size $\Delta$
for $\mathrm{j}:=0$ to XMAX step size $\Delta$
count:=0
for $\mathrm{k}:=0$ to num_not_charged_yet
if distance (i,j,
nodes[uncharged_nodes [k]][0],
nodes[uncharged_nodes
$[\mathrm{k}]][1])<=\mathrm{R}$
count ++
if count $>$ high_freq
high_freq:=count
high_freq_x:=j
high_freq_y:=i

//Here, we will find the highest frequency point in the //grid that will be the point, where we need to place //our first charging node to charge all the nodes in the $/ /$ network.

$\mathrm{i}:=0$

for $\mathrm{j}:=0$ to num_not_charged_yet

if distance(high_freq_x, high_freq_y, nodes[uncharged_nodes[j]][0],

nodes[uncharged_nodes[j]][1])>R temp $[\mathrm{i}++]:=$ uncharged_nodes $[\mathrm{j}]$

for $\mathrm{j}:=0$ to $\mathrm{i}$ uncharged_nodes[j]:=temp[j]

Figure 3. Grid Approximation algorithm

\section{Complexity Analysis}

Here, we assume that the Dimension or Grid size, $\mathrm{G}=\mathrm{M} * \mathrm{M}$, Number of sensors $=\mathrm{N}, \mathrm{C}_{\mathrm{D}}=$ Cost for calculating distance, $\mathrm{C}_{\mathrm{G}}=$ Cost of each comparison. As mentioned above, we can say that there are mainly two steps in this algorithm. In the first step, we have to find out the complexity intended for finding the distance of all sensor nodes from all grid points. Complexity in this step is $\mathrm{GN}_{\mathrm{D}}$. In second step, the algorithm will go by finding the point that will cover the maximum number of sensing nodes, for each comparison, and the complexity is $\mathrm{GC}_{\mathrm{G}}$. These steps must be regulated for each sensor nodes or grid points. So, In Worst case, the complexity is: $\mathbf{G}\left(\mathbf{N ~ C}_{\mathbf{D}}+\mathbf{C}_{\mathbf{G}}\right) * \mathbf{m i n}(\mathbf{G}, \mathbf{N})$. In Best case, the complexity is: $\mathbf{G}\left(\mathbf{N ~ C}_{\mathbf{D}}+\mathbf{C}_{\mathbf{G}}\right)$. In Average case, the complexity is: $1 / 2 * \mathrm{G}(\mathrm{N}$ $\left.\mathrm{C}_{\mathrm{D}}+\mathrm{C}_{\mathrm{G}}\right)^{*} \min (\mathrm{G}, \mathrm{N})=1 / 2 * \mathrm{G}\left(\mathrm{NP} \mathrm{C}_{\mathrm{G}}+\mathrm{C}_{\mathrm{G}}\right) * \min (\mathrm{G}, \mathrm{N})$ [using $\mathrm{C}_{\mathrm{D}}=\mathrm{PC}_{\mathrm{G}}$, (where $\mathrm{P}$ is a given value)], so the average complexity finally is $1 / 2 * \mathbf{G C}_{\mathbf{G}}(\mathbf{N P}+\mathbf{1}) * \min (\mathbf{G}, \mathbf{N})$.

\section{EXPERIMENTAL RESULTS}

In this section, we apply the approximation algorithm on various network topologies to demonstrate the algorithm's efficiency. Different experimental results have shown here by verifying different parameters of the network. In our work, the changing parameters for better understanding the scenario are transmission range R, i.e., a sensor's covering range of receiving and transmitting signals, number of sensing nodes $\mathrm{N}$ and total networking area or dimension, D. As we have deployed the sensors randomly in the network, for having the best result and gain the accuracy, we have taken the same snapshot of experimental result for five times and finally have taken the average.

We have showed our experimental results in three charts by verifying the concerning parameters which has shown in Fig. 4, 5 and 6.In these figures we consider the distance between the grid points to be unit and have showed the other parameters behavior to better understand the scenario. In Fig. 7, 8 and 9 we have shown the impacts of changing the different distances between the grid points. In Fig. 4, we have shown the result for the dimension 50*50, whereas in Fig. 5 and Fig. 6 that value will be changed to $150 * 150$ and $200 * 200$. The number of nodes used in this paper differs from 50 to 300 and if necessary can be expanded as well. While taking different values of transmission range we would prefer to maintain a margin in low, medium and high range of measurement, and so, we preferred to take the values as 25, 40 and 70 in Fig. 4, 5 and 6.

Here, we can see that for different dimensions and number of nodes, when the radius or transmission range of the nodes increases, the amount of charger requires decreases. This is because, with increasing radius more nodes can be in the range of the grid points (i.e., by placing a charger at that point we can charge all the sensors that are within the transmission range of the point) and number of charger is lessening accordingly. Here, it can be noted that the charger required is minimum for highest valued transmission range, i.e., 70 in every charts and lowest for the low valued transmission range, 25. 


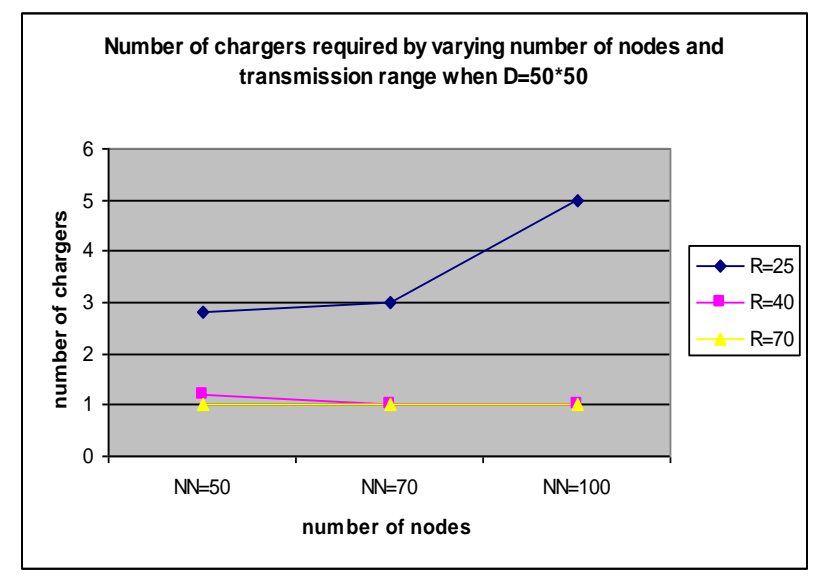

Figure 4. Results by changing transmission range and number of nodes for dimension 50

Another important parameter of the network is number of sensing nodes, N. In most of the cases, we need a sensor network with maximum number of nodes deployed in the network for establishing WSNs different kinds of applications. If we have a look in the Fig. 4, 5 and 6, we can see that, in most of the cases, as number of sensing nodes increase the number of chargers require increase accordingly. This is because, with higher number of nodes, more charging nodes are necessary to embrace them meeting the corresponding criteria.

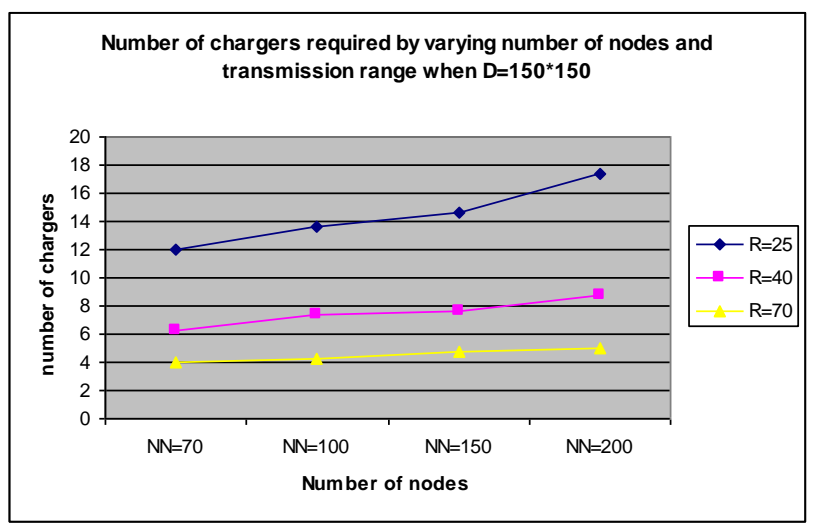

Figure 5. Results by changing transmission range and number of nodes for dimension 150

A last concerning criterion of the network is its dimension, D. In a large area, where nodes are placed randomly it is normally happens that they are placed in a scattered manner and that's why more chargers are required to charge all the sensing nodes in the network for such a network. The Fig. 4 is designed for low sized area network, 50*50, whereas in Fig. 5 and 6 we take different results for the area $150 * 150$ and $200 * 200$ accordingly. In Fig. 4, with the transmission range 25 and number of sensing nodes 100 , we can see that the numbers of chargers required are approximately 5. Whereas, for the same parameters in Fig. 5, which is designed for dimension
150, the chargers required are approximately 13 and in Fig. 6 it is 18 for dimension 200 .

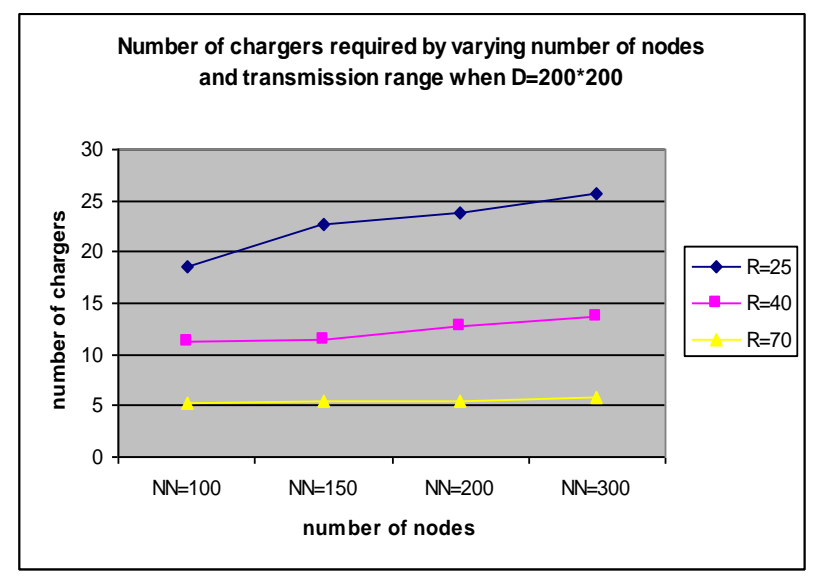

Figure 6. Results by changing transmission range and number of nodes for dimension 200

Now, we will discuss about changing the step size, i.e., the distance between the grid points that have shown in Fig. 7 and 8. In Fig. 7, we have shown the result for different number of sensing nodes, whereas in Fig. 8, we have changed the values of transmission range to show different results. We can see in Fig. 7, the value of $\mathrm{R}$ has taken 20 here, so the highest value of the step size as stated before is $\sqrt{2} * \mathrm{R}$, means $\sqrt{2} * 20$, i.e., 28 . Here, we can see that for different step sized value, as the distance between the grid points increase, the amount of chargers needed also increase. The reason is, as the distances between the grid points increase, the grid points are placing in far distances than previous unit distanced grid point placement and their searching area also increase accordingly, whereas, in lower step size valued grid, the more grid points are placed in close distance and checked accordingly and thus the number of chargers required are minimum for such a scenario. Moreover, to gain the efficiency, processing steps and time need to be low and for this reason it is necessary to increase the step size of the grid approximation.

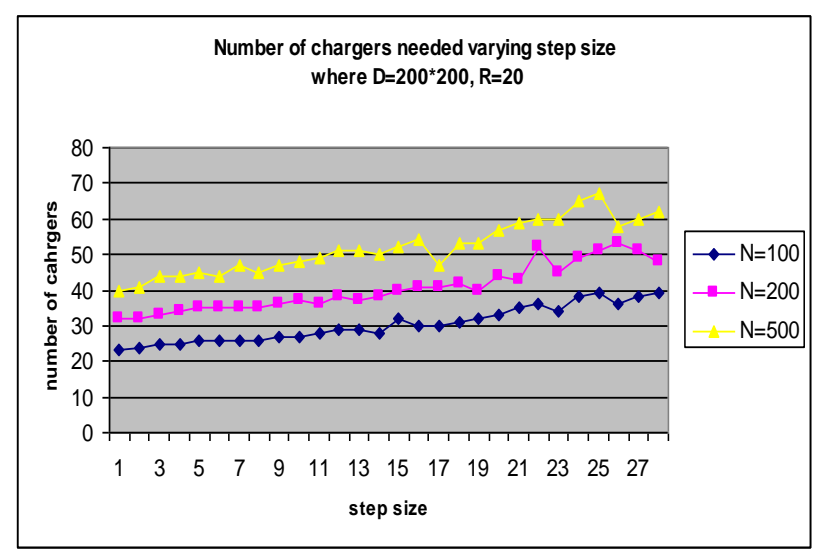

Figure 7. Results for different step size value by changing $\mathrm{N}$ and keeping $\mathrm{D}$ and $\mathrm{R}$ fixed. 
In Fig. 8, we have taken same consideration for different transmission range and here, we have kept the number of nodes to fix. Here, we can see that for higher valued transmission range, the numbers of chargers needed are low compare to the lower valued transmission range. The reason behind this is same as stated before for Fig. 4, 5 and 6 .

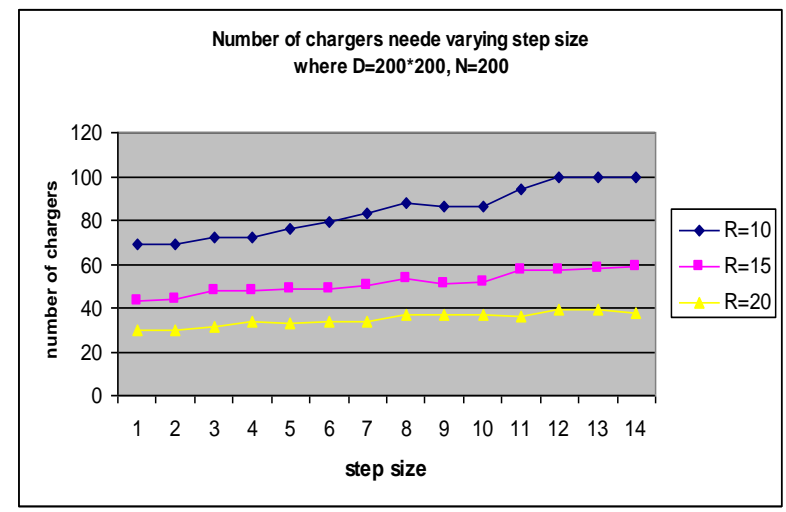

Figure 8. Results for different step size value by changing $R$ and keeping $D$ and $\mathrm{N}$ fixed.

\section{CONCLUSION AND FUTURE WORK}

Limitation of continuous energy or power supply is a prime concern in Wireless Sensor Networks (WSNs). To meet different applications of WSNs, it is very important for the sensors to have the longer lifespan. In this paper, we propose a solution to power limitation problem of WSN by an inductive charger deployment scheme to charge all the sensing nodes in the network using inductive charging technique. Here, we present a grid approximation algorithm to find out a deployment strategy for chargers with the goal to achieve the cost effectiveness and efficiency. The proposed algorithm will find out the location as well as the minimum number of chargers needed to charge all the sensing nodes in the network in order to maintain a continuous energy supply in the network which will help a sensor network to meet its various applications in an unfriendly environment. Moreover, the algorithm is designed for different step sized valued grid approximation and hence ensure the reduction of processing steps and time to make the algorithm much stronger and flexible. Different experimental results by changing different parameters have shown the strength of the algorithm for the proposed scheme.

As an advance to our work, in future, we have desire to work on different deployment approaches by developing more strong and innovative algorithms to solve the energy limitation problem of WSNs. Moreover, as our proposed algorithm is a generalized one, we have plan to expand our idea in the field of security for calculating minimum number of malicious nodes necessary to corrupt or jam the overall network and with this regard to measure a network strength of security. Moreover, we have aim to explore some more methodologies to implement the concept of this paper in real world and also explore for intelligent agent based deployment policies to achieve the goals.

\section{REFERENCES}

[1] A. Azim and M. M. Islam, "Hybrid LEACH: A relay node base low energy adaptive clustering hierarchy for wireless sensor networks", IEEE $9^{\text {th }}$ Malaysia International Conference on Communications (MICC), pp.911-916, 2009.

[2] A. Azim and M. M. Islam, "Dynamic service policy-based clustered wireless sensor networks", IEEE $6^{\text {th }}$ International Conference on Wireless and Mobile Computing, Networking and Communications (WiMob), pp. 196-202, 2010.

[3] A. Azim and M. M. Islam, "A dynamic round-time based fixed low energy adaptive clustering hierarchy for wireless sensor networks", IEEE $9^{\text {th }}$ Malaysia International Conference on Communications (MICC), pp.922-926, 2009.

[4] W. Yao, M. Li and M-Y Wu, "Inductive charging with multiple charger nodes in wireless sensor networks", APWeb Workshops, LNCS 3842, pp 262-270, 2006.

[5] Chevalerias, O. O. Donnell, T. Power, D. O Donovan, N. Duffy, G. Grant and G. O Mathuna, "Inductive telemetry of multiple sensor modules", Pervasive Computing, Vol. 4, pp. 46- 52, 2005.

[6] A. LaMarca, D. Koizumi, M. Lease, S. Sigurdsson, G. Borriello, W. Brunette, K. Sikorski and D. Fox, "Making sensor networks practical with robots," Lecture Notes on Computer Science (LNCS) 2414, pp. 152-166, 2002.

[7] What is Inductive Charging, http://www.wisegeek.com/what-isinductive-charging.htm, last visited: 05 January 2011.

[8] Splashpower Wireless Power in now eCoupled, http://www.splashpower.com, last visited: 05 January 2011.

[9] J. M. Kahn, R. H. Katz and K. S. J. Pister. "Next century challenges: mobile networking for smart dust", Proc. MobiCom, pp. 271-278, 1999.

[10] J. Paradiso, M. Feldmeier, "A Compact, Wireless, Self-Powered Pushbutton Controller", Proc. International Conference on Ubiquitous Computing, pp. 299-304, 2001.

[11] S. Meninger, J. O. Mur-Mirands, R. Amirtharajah, A. P. Chandrakasan and J. H. Lang, "Vibration-to-electric energy conversion", Proc. ACM/IEEE International Symposium on Low Power Electronics and Design, pp. 48-53, 1999.

[12] F. GaoJun and J. ShiYao, "Coverage Problem in Wireless Sensor Network: A Survey", Journal of Networks, Vol.5, No.9, September 2010.

[13] Ramachandran, "Real-atom grid approximation (RAGA) a new technique of crystal structure analysis using only amplitudes without determination of phases of reflections", Acta Crystallographica, Section A, Foundations of Crystallography, 46 (5). Pp 359-365.

[14] Minimum Set Cover, http://www.sprklab.com/notes/16-minimum-setcover/, last visited: 04 January 2011.

[15] Greedy Approach, http://ist.marshall.edu/ist238/greedy_appr.html, last visited: 04 January 2011.

[16] M. Cardei and D. -Z. Du, "Improving wireless sensor network lifetime through power aware organization”, Wireless Networks 11, pp. 333-340, 2005.

[17] F. Ye, G. Zhong, S. Lu and L. Zhang, "Energy efficient robust sensing coverage in large sensor networks", Technical Report ULCA, 2002.

[18] M. Cardei and J. Wu, "Coverage in wireless sensor networks", Department of Computer Science and Engineering, Florida Atlantic University, Boca Raton, FL 33431, www.cse.fau.edu/ jie/coverage.ps, last visited: 05 January 2011.

[19] S. Slijepcevic and M. Potkonjak, "Power efficient organization of wireless sensor networks", Proc. of IEEE International Conference on Communications, Vol-2, pp 472-476, Helsinki, Finland, June 2001. 
[20] M. Cardei, D. MacCallum, X. Cheng, M. Min, X. Jia, D. Li and D.Z.Du, "Wireless sensor network with energy Efficient Organization", Journal of Interconnection Networks, Vol 3, No 3-4, pp 213-229, Dec 2002.

[21] M. Cardei and D. -Z. Du, "Improving wireless sensor network lifetime through power aware organization", in press.

[22] M. Cardei, M. T. Thai, Y. Li and W. Wu, "Energy-Efficient target coverage in wireless sensor networks", In Proc. of INFOCOM, pp.19761984, 2005

[23] Pythagoras Theorem, http://www.mathsisfun.com/pythagoras.html, last visited: 05 January, 2011

\section{AUTHORS PROFILE}

Fariha Tasmin Jaigirdar is working in Stamford University, Bangladesh as a Lecturer in the dept. of Computer Science. She has completed her Bsc Engineering from Chittagong University of Engineering and Technology
(CUET) securing $5^{\text {th }}$ position in merit list. Now She is doing her Msc in Bangladesh University of Engineering \& Technology (BUET). She is young, energatic and hard working. Her research interest includes wireless networks, digital signal processing and bangla language display in digital form. Recently she is working on various research works to prove her best with her academic excellence as well as her analytical ability.

Mohammad Mahfuzul Islam is working in Banglades University of Engineering \& Technology (BUET) as an Associate Professor in the dept. of Computer Science \& Engineering. He has completed his Phd from Australia. $\mathrm{He}$ has a huge amount of publications in internationala journals and conferences. His research interest is wireless resource management, network security, image processing and artificial intelligence.

Sikder Rezwanul Huq is working in Stamford University Bangladesh as a Lecturer in the dept. of Computer Science. He has completed his Bsc Engineering from Islamic University of Technology (IUT). His research interest is grid computing and wireless networks. 